3 Jackson K, Butler R, Aujayeb A. Lung ultrasound in the COVID-19 pandemic. Postgraduate Medical Journal 2020 [Epub ahead of print]

4 Rajamani A, Shetty K, Parmar J et al. Longitudinal competence programs for basic point-of-care ultrasound in critical care: a systematic review. Chest 2020;158:1079-89.

5 Alber KF, Dachsel M, Gilmore A et al. Focused acute medicine ultrasound (FAMUS). Acute Med 2018;17:164-7.

\section{Nosocomial COVID-19 on a green ward}

DOI: $10.7861 /$ clinmed.Let.20.6.8

Editor - We read the recent article on nosocomial spread of COVID-19 on a stroke/neurology ward with interest. ${ }^{1}$ We did a similar observational study in our hospital during the peak of the pandemic. The stroke ward was re-designated as a 'green ward', where patients with acute stroke and with non-COVID-19 symptoms were admitted. We wanted to estimate the risk of nosocomial COVID-19 because there was a perception that the risk of COVID-19 was negligible on a green ward.

During the study, we included all patients who stayed for more than 7 days from 15 March 2020 to 30 June 2020. We chose 7 days because, if patients developed COVID-19 after 7 days, we could be certain that they contracted the disease while they were an inpatient rather than delayed diagnosis from admission. Patients were tested for COVID-19 if they developed symptoms, had contact with a symptomatic patient or before transfer to the community. During the study period, staff were advised to use a surgical mask, apron and shield as per public health guidelines. ${ }^{2}$

Four-hundred and forty-three patients were admitted to the green ward during the study period; 138 patients stayed for more than 7 days, of which 59 tested positive either while as an inpatient or within 7 days of discharge and 12 patients died. During the same period 27 out of 47 healthcare professionals contracted COVID-19.

Despite using the recommended PPE, $42 \%$ of patients who stayed more than 7 days contracted COVID-19, of whom 21\% died. Fiftyseven per cent of healthcare professionals developed COVID-19, of which, one needed respiratory support and all of them recovered.

Our figures are probably an underestimate due to the low sensitivity of the oropharyngeal swab (60\%) and as we did not test all patients and staff, we might have missed a few asymptomatic cases. ${ }^{3}$

In summary, the risk of nosocomial COVID-19 is high if patients stayed in for more than 7 days and the risk to healthcare workers is extremely high, even on a green ward. This suggest that the recommended protective measures taken to prevent nosocomial COVID-19 were inadequate.

WIN MAR SOE Core medical trainee, Glan Clwyd Hospital, Rhyl, UK

ASWATHI BALAKRISHNAN Core medical trainee, Glan Clwyd Hospital, Rhyl, UK VEDAMURTHY ADHIYAMAN Consultant geriatrician, Glan Clwyd Hospital, Rhyl, UK

\section{References}

1 Jewkes SV, Zhang Y, Nicholl DJ. Nosocomial spread of COVID-19: lessons learned from an audit on a stroke/neurology ward in a UK district general hospital. Clin Med 2020;20:e173-7.

2 Public Health England. Recommended PPE for healthcare workers by secondary care inpatient clinical setting. NHS and independent sector. PHE, 2020. https://assets.publishing.service.gov.uk/ government/uploads/system/uploads/attachment_data/ file/886707/T1_poster_Recommended_PPE_for_healthcare _ workers_by_secondary_care_clinical_context.pdf

3 Centre for Evidence-Based Medicine. Comparative accuracy of oropharyngeal and nasopharyngeal swabs for diagnosis of COVID-19. CEBM, 2020. www.cebm.net/covid-19/comparative-accuracy-oforopharyngeal-and-nasopharyngeal-swabs-for-diagnosis-of-covid-19

\section{Vitamin D deficiency and COVID-19}

\section{DOI: 10.7861/clinmed.Let.20.6.9}

Editor - Weir and colleagues timidly state that 'If vitamin D does in fact reduce the severity of COVID-19 ... [then] supplements would offer a relatively easy option to decrease the impact of the pandemic." This reticence ignores about 40 years of developing scientific knowledge of the pivotal role of vitamin D, and its many unique extra-endocrine functions, including in the primary immune process and down-regulation of inflammation in all known infections, including ones new to man. ${ }^{2}$ It is a vital nutrient, formed uniquely by action of UVB light on 7-dehydrocholesterol in the skin, when the sun is high overhead. In the light of variations in latitude, seasons, weather, skin colour, clothing cover, use of sun blocks and indoor living, the important question is not whether it should be taken, but how much. This can only be determined by measuring blood vitamin D levels, although the mantra of supposed overdose risk is grossly exaggerated.

There are now, to our knowledge, 14 studies that indicate the specific benefit in the COVID-19 pandemic of having a blood level of vitamin $D$ greater than $30 \mathrm{ng} / \mathrm{mL}$ ( $75 \mathrm{nmol} / \mathrm{L}$ ), and a very significant danger of death from infection if the blood level lies below $10 \mathrm{ng} / \mathrm{mL}$ ( $25 \mathrm{nmol} / \mathrm{L}$ ). ${ }^{3}$ We would not dream of treating diabetes without a knowledge of the blood glucose level. Likewise, with vitamin $D$ deficiency at a time of a serious pandemic, the target blood level is critical, should be checked for and the dose adjusted accordingly. We suggest a target level of not less than $30 \mathrm{ng} / \mathrm{mL}$ and not more than $60 \mathrm{ng} / \mathrm{mL}$. In most adults, this will be achieved by a mean D3 supplement of 4,000 IU daily. This is incidentally close to the recommended daily intake, weight-forweight, for an adult laboratory mouse ( 100 IU/kg). ${ }^{4}$

Another neglected fact is that vitamin D, acting via its VDR receptor, forms the working heterodimer not alone but paired with the vitamin A-activated retinol receptor (RXR). Yet National Institute for Health and Care Excellence (NICE) guidelines are dismissive of vitamin D's role in immunity, while generous in that of vitamin $A{ }^{5}$ This is completely illogical as, in their joint functions, the two match each other mol for mol.

NICE is a body that is orientated towards evaluating randomised controlled trials (RCTs) of pharmaceutical products, and so largely discounts observational studies. It does not appear to understand biological systems, such as the case of vitamin D, where sufficiency of an inactive natural storage precursor is crucial for the production of an active one that has many other local roles, in addition to the obvious general control of calcium absorption and bone health. ${ }^{6}$

But to help satisfy NICE skeptics, fortunately we now have an RCT from Córdoba, Spain, into the effect of vitamin D in COVID-19 pneumonia, which appeared after the paper by Weir and colleagues. ${ }^{7}$ It demonstrated that, in those treated with oral $25(\mathrm{OH}) \mathrm{D}$, there was a subsequent need for intensive care unit transfer in only $2 \%$ (with no deaths), versus $50 \%$ and two deaths in the control group. Evidence is cumulative, and is about more than RCTs, as Sir Austin Bradford 